\title{
Méthode d'étude de la circulation intra-osseuse chez l'homme et chez l'animal
}

\author{
A method for investigation of human \\ and animal intra-osseous circulation
}

\author{
Jacques Arlet \\ Professeur de Rhumatologie
}

\author{
Jean-Pierre Géral \\ Professeur de Physiologie
}

\author{
Paul Ficat \\ Professeur de Chirurgie Orthopédique
}

\section{Introduction}

L'os a été trop longtemps considéré comme une armature plus ou moins inerte et sa circulation négligée. En réalité, sa vitalité, sa puissance de renouvellement est éclatante quand on observe la réparation souvent totale et parfaite d'une fracture.

L'os n'est jamais en repos, il se renouvelle tout au long de l'existence et selon un programme imprimé dans ses cellules mêmes et répondant à des nécessités mécaniques. De plus, il a, au moins, deux autres responsabilités majeures :

1) celle d'assurer le stockage et la libération immédiate ou retardée du calcium nécessaire à l'équilibre ionique du plasma et à la charge électrique des membranes cellulaires;

2) celle d'assurer le renouvellement des cellules $d u$ sang, globules rouges et polynucléaires en particulier.

On comprend alors pourquoi la circulation intraosseuse est aussi nécessaire à la vie de l'os que la circulation cérébrale à la vie du cerveau.

Si les médecins ont tardé à réaliser cette évidence, c'est qu'ils n'avaient pas les moyens d'explorer la circulation intra-osseuse. Il est vrai que cette exploration est difficile à cause des particularités de cette circulation. Ces particularités tiennent à 2 faits anatomiques simples :

1) La multiplicité des voies d'entrée et de sortie du sang pour un os donné. Tout le sang d'un rein arrive par une seule artère rénale, il est facile de mesurer et de contrôler les entrées dans un tel organe. Rien de tel n'est possible pour un os qui reçoit 6 groupes d'artères, d'où la difficulté d'en apprécier le débit (Fig. 1);

2) Un lit vasculaire intra-osseux enfermé dans une cavité à parois rigides et indéformables. Ses changements de volume nécessaires en fonction de besoins variables ne sont supportables que si le tissu de remplissage,

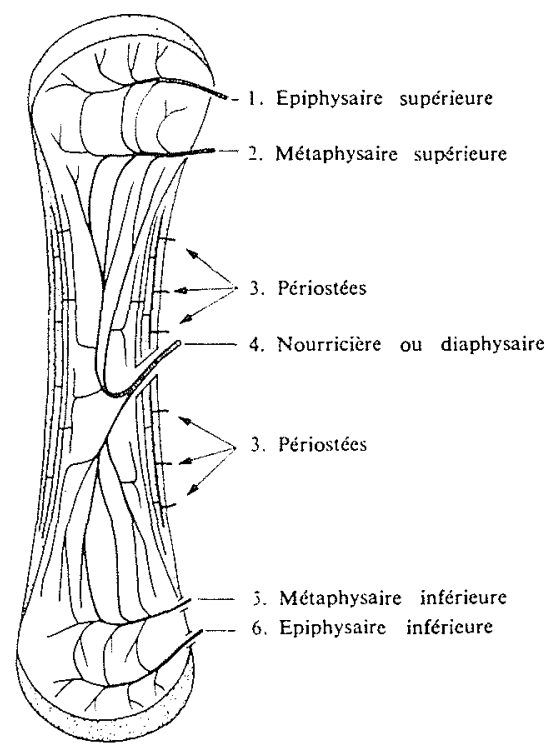

Figure 1 - Schéma de la vascularisation artérielle d'un os long avec ses six groupes d'artères.

essentiellement graisseux, adapte lui-même rapidement son volume en fonction des impératifs de la circulation. C'est le réseau capillaire (intermédiaire entre artères et veines) qui s'aplatit (capillaires dormants) ou se dilate, ralentit ou accélère sa prolifération selon les besoins. Il s'agit d'une régulation permanente et sectorisée qui dépend des hormones vaso-actives et des nerfs vasomoteurs.

On comprend facilement qu'elle est difficile à étudier à travers une coque aussi dure et aussi épaisse, opaque aux rayons $X$ et qu'elle puisse se dérégler assez facilement, laissant se développer, en particulier, un excès de pression intra-osseuse, une hyperpression qui bloque et gèle la micro-circulation. 
Depuis 15 ans, les efforts de notre équipe ont d'abord été appliqués à la recherche de moyens d'exploration de la circulation intra-osseuse afin de mettre à la disposition des médecins des moyens simples pour déceler les maladies vasculaires de l'os. Dans le même temps, on pouvait espérer, à l'aide de ces méthodes, accroitre nos connaissances de la physiologie normale de la circulation osseuse.

\section{Méthodes proposées}

Le premier groupe correspond à la visualisation directe des vaisseaux destinés à l'os ou situés dans l'os et cela dans des conditions vitales. Nous dirons un mot des trois méthodes actuellement à notre disposition : artériographie, veinographie, cinématographie des capillaires.

L'injection dans une artère d'un liquide opaque aux rayons $\mathrm{X}$ (produit iodé) permet de suivre le trajet de ses branches, leur morphologie et leur distribution; c'est l'artériographie, qui a déja rendu d'inestimables services au diagnostic médical pour visualiser en particulier les artères des membres et les artères cérébrales.

Il y a 20 ans, on pensait qu'il était impossible de voir de cette façon, la pénétration dans l'os des artères à destinée osseuse. Actuellement, on sait le faire en injectant le produit opaque près de l'os à l'aide d'une sonde très fine (cathéter) que l'on place "sélectivement" à l'entrée de l'artère que l'on veut voir. Cette artériographie "sélective" a permis en particulier de visualiser les branches terminales intra-osseuses de l'artère épiphysaire supérieure de la tête fémorale (Fig. 2).

La veinographie de l'os est appelée phlébographie per osseuse ou trans-osseuse : si l'on injecte un liquide dans la moëlle osseuse, il pénètre rapidement dans les capillaires et il est évacué de l'os par les veines de drainage. Si le liquide injecté est opaque aux rayons $X$, il dessine le réseau capillaire à l'endroit de l'injection puis les veines qui s'échappent de l'os : l'évacuation est rapide, les veines extra-osseuses commencent à être visibles en moins d'une seconde et la totalité du produit injecté a quitté l'os en 1 à 2 minutes. On peut cinématographier le phénomène ou prendre des clichés en série qui permettent de mesurer la vitesse de drainage et de découvrir dans les cas pathologiques, un ralentissement du drainage, voire une stase intra-osseuse de plus ou moins longue durée (Fig. 3 et 4 ).

Quant à la cinématographie directe du lit capillaire, elle a été réalisée par un médecin suédois (Branemark) qui, après trépanation du cortex osseux, place l'objectif d'une caméra dans cette fenêtre de trépanation et filme les capillaires et la circulation des globules du sang à l'intérieur des capillaires. Il a pu ainsi confirmer la variabilité du lit capillaire et l'existence de voies de dérivation, de court-circuits directs entre circulation veineuse et circulation artérielle, permettant d'éviter les "encombrements". Pour le moment, cette technique n'a été utilisée que chez le lapin.

Une autre méthode intéressante d'exploration de la circulation osseuse est la thermométrie, mise au point à Charleroy par Raynal et Lévy. Elle consiste à placer

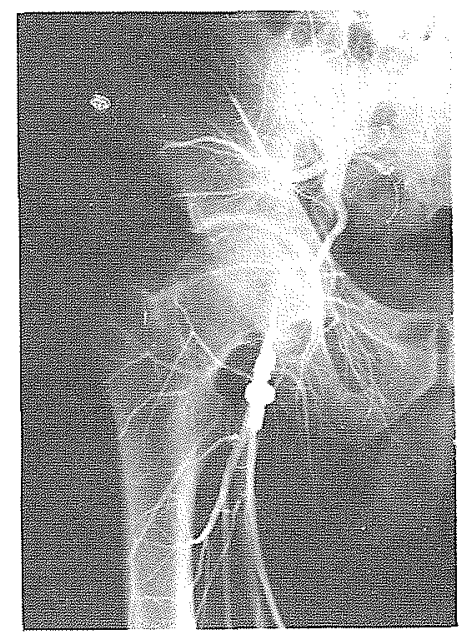

Figure 2 - Artériographie sélective de la hanche.

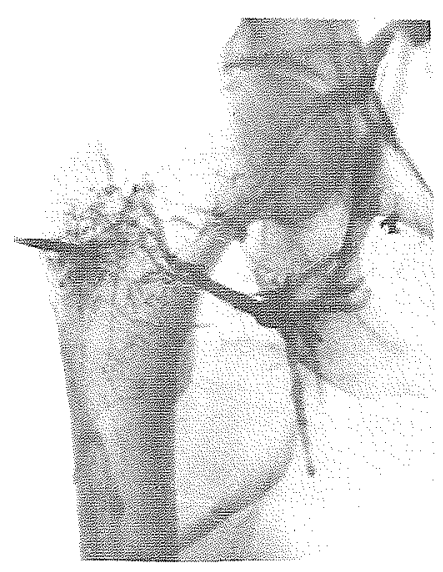

Figure 3 -Phlébographie per osseuse normale. L'injection d'iode a été faite à l'intérieur de l'os. La radiographie a été prise à la fin de l'injection. On voit le réseau capillaire et veineux intra-osseux et surtout le remplissage immédiat des grosses veines de drainage qui sortent de l'os.

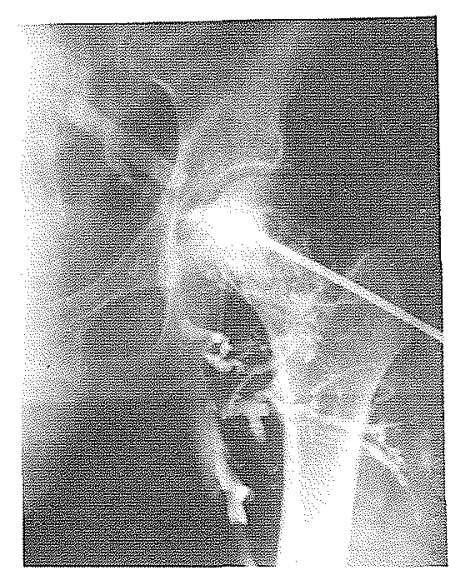

Figure 4 - Phlébographie per-osseuse anormale. L'injection a été faite au centre de la tête fémorale. La radiographie a été prise $5 \mathrm{mn}$ après la fin de l'injection : on voit encore une flaque de produit opaque au centre de la tête fémorale, ce qui traduit une stase anormale. 
dans l'os une sonde thermométrique (sonde à thermistance) étalonnée par rapport à une sonde rectale et reliée à un pont de Wheatstone. Sa sensibilité est de $1 / 10 \mathrm{de}$ degré. La température locale s'abaisșe quand le flux. sanguin diminue et là ou il est diminué, comme dans les foyers de nécrose de la tête fémorale.

C'est de l'étude de la pression intra-osseuse et du débit du sang osseux que nous voulons surtout parler.

\section{Pression intra-médullaire (P.I.M.)}

Quand on enfonce une aiguille reliée à un manomètre dans la moële osseuse d'un os long humain, on enregistre une pression positive, supérieure à la pression atmosphérique (considérée en l'occurence comme le point zéro de référence) de l'ordre de $20 \mathrm{~mm} \mathrm{de} \mathrm{Hg}$. Ainsi, l'os est "sous pression" comme un pneu est maintenu gonflé par l'hyperpression qui règne dans la chambre à air. Cette hyperpression contribue probablement à accroitre la résistance de l'os (Fig. 5).

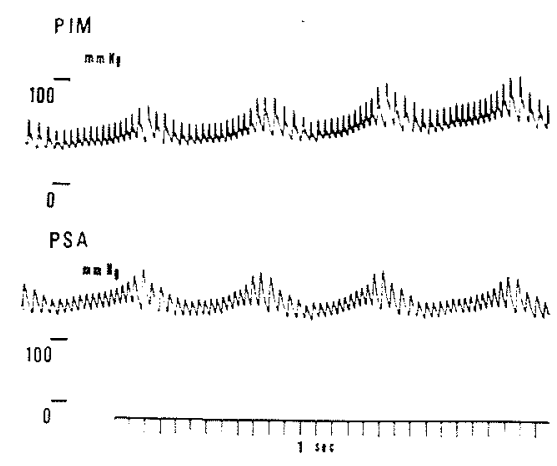

Figure 5 - Enregistrement simultané de la pression intramédullaire ou P.I.M. (tracé supérieur) et de la pression artérielle générale dans l'artère humérale ou P.S.A. (tracé inférieur). L'ondulation périodique de la courbe correspond aux mouvements respiratoires.

La moëlle osseuse est limitée par une membrane qui la sépare des structures dures, minéralisées de l'os. Cette membrane n'est pas imperméable (cela n'existe pas dans un organisme vivant) et elle est traversée par de fins vaisseaux qui relient la circulation médullaire à la circulation intra-corticale et a la circulation périostée extra osseuse, mais elle constitue un espèce de sac qui assure la cohésion de cette partie molle de l'os qu'est le tissu médullaire.

Plusieurs auteurs (Held et Thron, Wilkes et Visscher) ont mesuré la pression du tissu médullaire en mettant un tonomètre à l'extérieur du sac médullaire et à son contact : ils ont trouvé une pression très voisine de celle que l'on mesure en enfonçant l'aiguille dans la moëlle. En pratique donc, on peut dire que mesurer la pression intra-médullaire équivaut à mesurer la pression tissulaire de la moelle osseuse.

\section{a) Chiffres normaux de P.I.M.}

Chez l'animal, (chien, chat, lapin) de très nombreuses mesures ont été faites dans les os longs (fémur, tibia, humérus), dans la diaphyse (partie centrale cylindrique de l'os) et dans les extrémités (épiphyses). Les chiffres observés sont très variés, si bien que certains auteurs ont été tentés de considérer que la mesure de la P.I.M. n'est pas fiable ou que ce paramètre est trop labile pour être utilisable.

En réalité, l'analyse des données permet d'affirmer les faits suivants :

- la stabilité de la courbe de pression obtenue au bout de moins de cinq minutes est ensuite parfaite pendant toute la durée de l'expérience (parfois plusieurs heures)

- l'histogramme de la répartition des valeurs de P.I.M. sur une série de chiens, réalise une courbe de Gauss témoignant de la réalité physiologique de ce paramètre

- les P.I.M. mesurées au même moment dans 2 os symétriques, sont très voisines, voire identiques

- la valeur moyenne de la P.I.M. est différente selon l'animal d'expérience, selon l'os exploré et selon la partie de l'os examinée. La P.I.M. n'est pas la même dans les différentes parties de l'os, ce qui témoigne d'une sectorisation de la circulation intra-osseuse.

Chez lhomme normal et dans les os longs, les mesures ont été moins nombreuses mais les écarts sont peu importants et les chiffres observés très proches les uns des autres. De plus, la P.I.M. est plus élevée dans les extrémités articulaires de l'os (épiphyses) que dans sa partie moyenne (diaphyse) (Fig. 6). L'étude statistique des valeurs que nous avons mesurées dans le fémur, permet de conclure que la valeur frontière entre le normal et le pathologique est à $30 \mathrm{~mm} / \mathrm{Hg}$ : au-dessus de ce chiffre, on peut parler d'hyperpression.

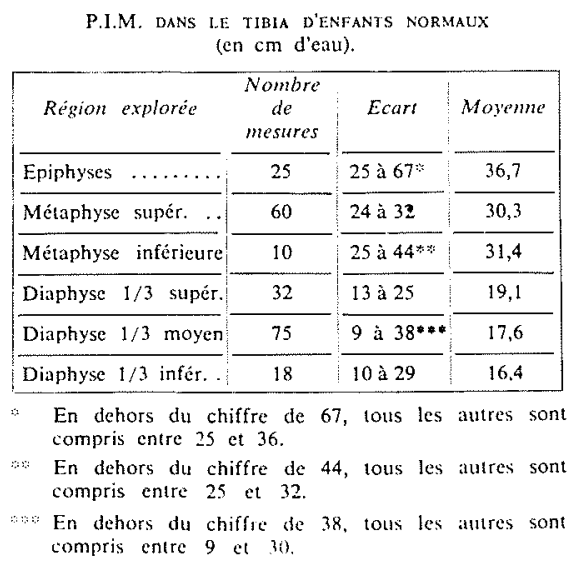

Figure 6 - Mesure de la pression intra-médullaire dans les tibias chez des enfants normaux (d'après Kabakele).

\section{b) Variations physiologiques spontanées de la P.I.M.}

- La courbe de pression enregistrée présente une ondulation régulière synchrone des battements cardiaques : c'est ce que l'on appelle le pouls osseux transmis au tissu médullaire par la pulsation des artères intraosseuses. Il y a donc une P.I.M. maxima et une P.I.M. minima avec un écart moyen de $4 \mathrm{~mm} \mathrm{Hg}$. Cette pulsa- 
tion intra-médullaire disparait si on ligature l'artère principale du membre chez l'animal ; chez l'homme, la compression digitale de l'artère fémorale supprime provisoirement la pulsation intra-fémorale (Fig. 7).
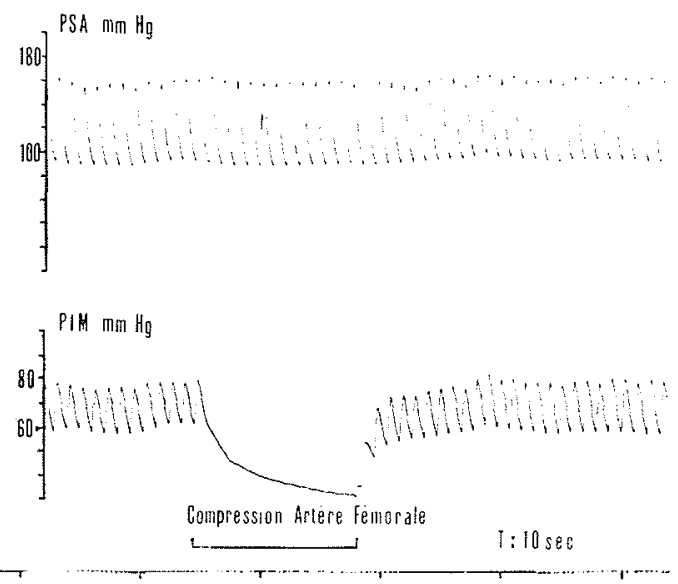

Figure 7 - Effet de l'occlusion provisoire de l'artère fémorale sur la pression intra-médullaire.

- La courbe de pression intra médullaire présente un deuxième type d'ondulation périodique en rapport avec la respiration : la P.I.M. diminue au cours de l'inspiration, elle augmente au cours de l'expiration. II en est de même de la pression veineuse et de la pression artérielle à l'extérieur de l'os (Fig. 5).

- Enfin il y a une double corrélation entre la pression artérielle générale (P.A.G.) et la P.I.M. : d'une part, toute modification brusque de la pression artérielle a un retentissement immédiat sur la P.I.M. ; d'autre part, chez l'homme nous avons constaté une relation statistique entre le niveau de la P.I.M. et le niveau de la P.A.G. Il ne semble pas toutefois que chez l'hypertendu, la P.I.M. dépasse $30 \mathrm{~mm} / \mathrm{Hg}$.

\section{c) Variations physiologiques provoquées de la P.I.M.}

- La contraction musculaire volontaire élève la P.I.M. Certains auteurs, finalistes, pensent que cela accroit la résistance mécanique de l'os au cours des efforts physiques.

- Si la compression de l'artère fémorale diminue la pression intra-médullaire dans le fémur et le tibia, à l'inverse l'occlusion de la veine fémorale élève la P.I.M. par stase intra-osseuse : cet accroissement simultané du volume du lit capillaire intra-osseux et de la pression intra-osseuse peut entraîner une gène circulatoire, puis une insuffisance circulatoire qui peut aller jusqu'à la mort de l'os.

Enfin, les hormones naturelles vaso-actives agissent sur la P.I.M. L'Adrénaline diminue la P.I.M., par suite de la vaso-constriction artériolaire qu'elle provoque. C'est probablement par cet intermédiaire que la stimulation électrique du sympathique réduit la P.I.M. (Fig. 8).

Par contre, la section du nerf principal du membre inférieur, le sciatique, entraine une augmentation de la P.I.M. comme on l'observe dans les os des poliomyélitiques du côté de la paralysie.

\section{Débit osseux}

Le débit sanguin d'un vaisseau est la quantité de sang qui s'écoule à travers la surface de section du vaisseau dans l'unité de temps ( 1 minute). Le débit d'un organe est la quantité de sang qui traverse cet organe en 1 minute. Le débit sanguin d'un os est, de la même façon, la quantité de sang qui le traverse en 1 minute ; comme c'est un organe rigide, inextensible, c'est aussi bien la quantité qui entre dans cet os en 1 minute que la quantité de sang qui en sort pendant le même temps.

\section{a) Méthodes de mesure}

Aucune n'est parfaite car il y a trop de vaisseaux qui entrent dans un os et en sortent, et qu'on ne peut pas tous les contrôler.

Il y a cependant un systeme de vaisseaux plus importants que les autres, les vaisseaux nourriciers : il y en a un ou deux par os, ce sont les plus gros, ils fournissent environ $50 \%$ du débit : les veines correspon-

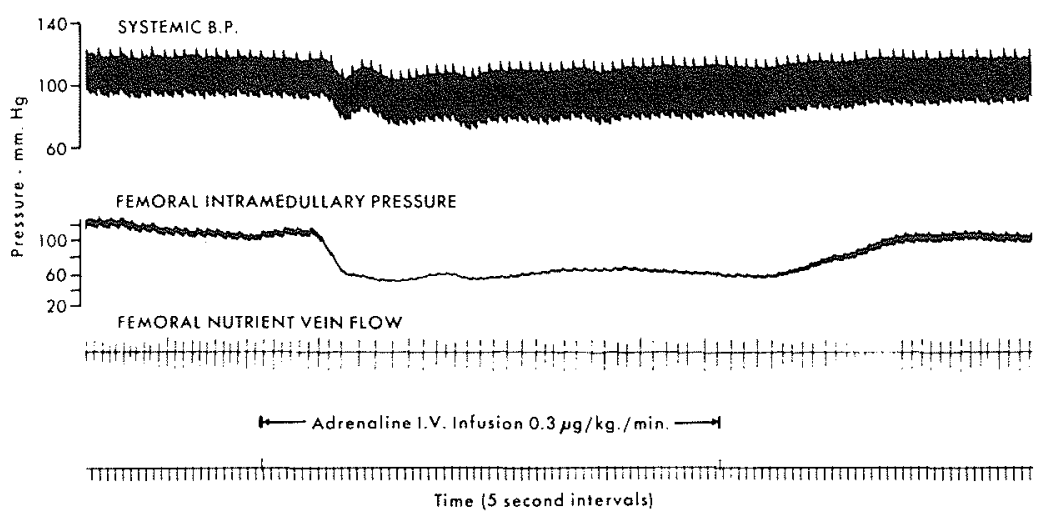

Figure 8 - Effet de l'injection d'Adrénaline (vaso-constructive) sur la pression intra-médullaire et le débit veineux de sortie (d'après Shim). 
dantes peuvent être cathétérisée chez le chien, leur sang recueilli sur un débitmetre compte goutte. Cette méthode ne permet pas de chiffrer avec exactitude le débit total d'un os mais elle sert à étudier les variations de débit sous diverses influences.

Seules les méthodes indirectes peuvent permettre de foumir une indication chiffrée du débit mais par un calcul approximatif. Les plus intéressantes sont les 3 suivantes; elles utilisent toutes les trois des corps radioactifs : mesure de la vitesse de disparition d'un produit radioactif injecté dans la moëlle osseuse; mesure de la radioactivité de l'os après injection dans la circulation artérielle de microsphères marquées qui se bloquent dans le système capillaire de l'os ; mesure de la radioactivité d'un os après injection dans la circulation générale d'un corps radio-actif qui se fixe électivement sur le tissu minéralisé (Bone seeking isotope) par exemple le Calcium 47 ou le Strontium 85.

Dans le premier cas, on utilise un corps radio-actif comme l'iode 131 ou le Tecnetium $99 \mathrm{~m}$, qui injecté dans la moëlle osseuse, pénètre immédiatement dans les capillaires veineux et s'évacue rapidement par les veines de drainage de l'os. Un compteur de Geiger placé audessus de la zone d'injection, permet de mesurer la décroissance de la radio-activité et de tracer une courbe de vitesse de disparition a partir de laquelle on calcule le débit pour $100 \mathrm{mg}$ d'os frais par minute. De toute façon, la courbe obtenue n'a de valeur que pour la zone injectée ; elle n'apprécie le débit que dans une région étroite de l'os. Cette méthode a cependant l'énorme avantage de pouvoir être utilisée facilement, sans danger ni douleur, chez l'homme. Elle nous aide a faire le diagnostic de ralentissement circulatoire dans la région étudiée.

Pour la deuxième méthode, on utilise des microsphères marquées au Technetium, injectées dans la circulation artérielle; elles se bloquent dans les capillaires. La radio-activité des différents organes et en particulier d'un os, est étudiée après sacrifice de l'animal et isolément de chaque os ou de chaque partie d'os. Cette méthode est précise mais n'a qu'un intérêt expérimental.

La troisième méthode utilise le Strontium 85 que l'os fixe, l'extrayant en quelque sorte du sang. Le Strontium est injecté dans la circulation générale, où sa concentration est connue et maintenue constante pendant 5 minutes. Au bout de ce temps, l'animal est sacrifié, l'os isolé, sa radio-activité mesurée. Le calcul du débit se fait selon l'équation de Fick :

$$
D(\text { débit })=\frac{Q \text { (quantité de } \mathrm{Sr} 85 \text { fixé sur l'os })}{A-\begin{array}{c}
V(\text { Différence artério-veineuse des } \\
\text { concentrations en Strontium })
\end{array}}
$$

Le calcul est approximatif car l'os ne fixe pas $100 \%$ du Strontium qui le traverse.

\section{b) Valeurs normales de débit osseux}

Les chiffres obtenus par les différents auteurs varient de 5 à 30 selon l'animal choisi et la méthode utilisée. II se situe en moyenne autour de $10 \mathrm{ml} / \mathrm{mn} / 100 \mathrm{gr}$ avec des différences locales d'un secteur de l'os à l'autre. C'est ainsi que le débit est d'autant plus élevé que l'on se situe dans une zone plus vascularisée; ce qui est normal.
Le débit du squelette dans son ensemble, est de l'ordre de $10 \%$ du débit cardiaque au repos, soit $500 \mathrm{ml} / \mathrm{mn}$.

\section{c) Contrôle du débit du sang osseux}

Trois groupes d'action contrôlent le débit osseux :

- Contrôle nerveux. La stimulation électrique des nerfs de l'os provoque une diminution du débit, leur section une augmentation. Cela signifie que le debit est contrôlé par les nerfs vaso-moteurs situés dans l'os autour des vaisseaux.

- Contrôle hormonal. L'Adrénaline réduit le débit intra-osseux (Fig. 8).

- Contrôle métabolique. Une déviation du $\mathrm{pH}$ sanguin du côté de l'acidité, par exemple par accumulation d'acide lactique ou de gaz carbonique, augmente le débit osseux. Il en est de même de l'hypoxie (baisse de la pression d'oxygène dans le sang).

\section{Commentaires}

1) Dans le domaine du diagnostic médical, certaines méthodes présentées ont déjà des applications formelles pour le diagnostic des troubles circulatoires intra-osseux. L'os subit, comme les autres organes, les conséquences d'une insuffisance circulatoire qui peut conduire à la nécrose, spécialement dans certaines régions où la circulation est moins richement anastomosée comme dans la tête fémorale. Les nécroses de la tête fémorale se voient dans des circonstances diverses, après fracture du col fémoral, chez les tubistes, chez les noirs atteints de drépanocytose, mais dans notre expérience, les cas les plus fréquents, ne relèvent pas de causes jusqu'ici connues. L'os nécrosé a le même aspect radiologique que l'os normal. Cette insuffisance de la radiologie pour le diagnostic précoce de nécrose osseuse, permet de comprendre l'intérêt des méthodes d'exploration dites fonctionnelles de la circulation osseuse et spécialement la mesure de la pression intra-médullaire, habituellement et parađoxalement trop élevée en cas d'ischémie et de nécrose osseuse (Fig. 9).

2) Dans le domaine de la physiologie et de la physiopathologie de la circulation intra-osseuse, l'étude des relations entre le débit intra-osseux et la pression intramédullaire est pleine d'enseignement.

La pression intra-médullaire est une pression tissulaire mesurée en aval des résistances artériolaires $(\mathrm{ra})$. Elle est beaucoup plus élevée que la pression dans les veines

Figure 9 - Diagramme de Pressions intra-médullaires mesurées au même endroit (spongieux trochantérien) dans 3 groupes de cas : nécroses osseuses, arthroses, hanches normales.

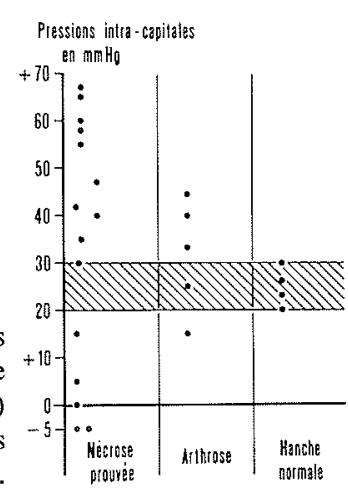


extra osseuses. Il faut donc qu'il existe à la sortie du lit capillaire une deuxième résistance du côté veineux $(r v)$. On sait que le débit est proportionnel à la pression et inversement proportionnel à la résistance, soit :

$$
\mathrm{D}=\frac{\mathrm{P}}{\mathrm{R}}=\frac{\text { P.S.A. }- \text { P.I.M. }}{r a}=\frac{\text { P.I.M. }- \text { P.V. }}{r \nu}
$$

L'existence de la résistance artériolaire est bien connue et sa régulation assurée par les nerfs vasoconstricteurs artériolaires qui augmentent $r a$ et diminuent $D$ et P.I.M. Sous leur action, débit et pression varient parallèlement.
Par contre, nous ne connaissons ni le siège exact de la résistance veineuse $(r v)$, ni les mécanismes de sa régulation. Cependant, sous l'effet de l'Adrénaline à faible dose, on peut observer une augmentation du débit intra-osseux, sans modification de la P.I.M., ce qui ne peut se faire sans diminution de la $r v$. De même dans les cas pathologiques, l'élévation excessive de la P.I.M. entraine une réduction du débit.

Ainsi l'étude des relations débit-pression est une des orientations les plus intéressantes dans les recherches futures en micro-circulation intra-osseuse.

\section{Discussion}

\section{Président : M. le Professeur R. COMOLET}

Le Président remercie le Professeur ARLET et M. GERAL pour leurs exposés et ouvre la discussion.

M. QUEMADA ayant demandé des éclaircissements sur la structure et sur le bilan de la circulation osseuse, le Professeur ARLET apporte les précisions suivantes:

Les artères et les veines ont des parois parfaitement définies, les capillaires aussi. A côté il y a des espaces sanguins où les parois sont moins nettement définies. Dans tout cela circule le sang. A travers les parois plus ou moins nettement définies passent les polynucléaires et les globules rouges. C'est d'ailleurs du sein même de ces cellules des capillaires que sort le sang: ces cellules se transforment en cellules sanguines. Les grosses cellules graisseuses que vous avez vues sur les clichés, qui sont volumineuses (de l'ordre de 25 à $75 \mu$ soit une moyenne de $50 \mu$ environ) ne sont absolument pas échangeables de l'intérieur à l'extérieur : elles se tiennent en permanence à l'extérieur de tout le système circulatoire. C'est simplement une question de volume réciproque. Il est certain que lorsque le volume des capillaires augmente, celui du tissu graisseux diminue. Il faut bien que cela fonctionne ainsi, sinon l'os éclaterait.

Sur une nouvelle question de M. QUEMADA, le Professeur ARLET précise qu'il ne s'agit pas de "globules graisseux" mais bien d'énormes cellules, avec des "globules à côté".

M. KOPP observe que le moelle osseuse n'étant certainement pas un fluide newtonien, elle est sans doute le siège de contraintes tangentielles ou normales qui se ne propagent pas de façon constante, puisqu'il n'y a pas écoulement; est-ce que ceci ne risque pas de provoquer certaines discordances entre la pression mesurée par un capteur externe et la situation des contraintes réelles à l'intérieur de l'os ?

Le Président relève l'importance de cette question de métrologie.

Ce qu'on mesure correspond-il à ce qui existe in situ ? résume-t-il en ajoutant... entre la partie artérielle et la partie veineuse il y a un hiatus, et ce hiatus c'est la moelle qui, selon les communications présentées, se trouve à une pression moyenne variant selon l'animal de 20 à $50 \mathrm{~mm}$ de mercure.

En réponse, M. GERAL se montre convaincu que l'on mesure bien une pression intra-osseuse même s'il peut paraître surprenant de trouver dans un tissu des pressions qui peuvent atteindre jusqu'à $90 \mathrm{~mm}$ de mercure.

Il y a à cela, ajoute-t-il, plusieurs raisons. D'abord, si l'on mesure la pression en divers points de l'os, on trouve la même pression en divers points de la moelle; il faudrait que l'on fasse exactement la même erreur de métrologie dans les deux cas.

D'autre part, on trouve la même pression et cela est très frappant - si l'on mesure directement la pression en faisant un trou dans l'os et en mesurant une pression veineuse terminale.

Reste à savoir ce que l'on mesure exactement. Je suis persuadé qu'il y a une pression dans la cavité médullaire. Il est évident qu'elle se situe après les résistances artérielles car, lorsqu'on contracte ces résistances, la pression intramédullaire chute. Mais je ne saurais pas en donner une localisation exacte sur notre schéma.

Je précise enfin que nous utilisons une aiguille de $1 \mathrm{~mm}$ de diamètre et que nous nous efforçons de léser la moelle le moins possible. Il n'y a en tout cas pas circulation de moelle dans l'ensemble capteur, tubulure, trocart, aiguille.

Répondant à une question du Président, M. GERAL indique que la seringue n'est pas remplie de sang mais d'un liquide salé isotonique empêchant la coagulation.

Il rappelle également que la moelle n'est pas un liquide mais une structure cellulaire constituant un tissu à la fois très friable, très mou et susceptible de conserver des gradients de pression très importants.

M. le Professeur LEMAIRE suggère de recourir aux électromanomètres miniaturisés maintenant utilisés pour l'exploration des très petites artères. Ainsi éviterait-on, pense-t-il, les incertitudes de la méthode du manomètre de compensation utilisée par les Suisses.

Le Docteur ARLET observe que l'une des difficultés de ces mesures est de savoir exactement où on se situe: dans un vaisseau? une artère? dans un capillaire? dans une veine?

La valeur de la méthode lui semble pourtant confirmée par le fait que les Suisses, ayant procédé à des mesures sans perforer la membrane séparant la moelle du cortex, ont trouvé les mêmes chiffres que lui : environ $20 \mathrm{~mm}$ d'hyperpression.

$M$. le Professeur LEMAIRE suggère d'opérer en deux temps : placer un trocart et contrôler la position par radioscopie, placer le capteur ensuite.

Le Docteur ARLET décrit sommairement, à la demande de M. BONIS, la structure de la moelle osseuse : c'est un tissu constitué essentiellement de trois éléments :

- l'ensemble des artères, des capillaires (assez nombreux) et des veines,

- un tissu entre les vaisseaux, avec des cellules hématopoïétiques, constituant la moelle active du point de vue du sang,

- un tissu graisseux de remplissage fait de grosses cellules rondes qui sont les lipocytes.

Le Président demande si ces vaisseaux d'évacuation du sang sont collabables.

Le Docteur ARLET confirme que les capillaires sont de dimensions très variables et que certains - appelés capillaires dormants - sont même complètement colabés.

Le Président émet l'hypothèse que ces capillaires plus ou moins écrasés soient à l'origine de la chute de pression de 50 à $5 \mathrm{~mm}$ constatée au niveau de la moelle.

Le Docteur ARLET et M. GERAL pensent que c'est une hypothèse intéressante. Ils font observer combien le fonctionnement de ces vaisseaux est particulier. La pression extérieure est à peu près uniforme et ils ont une pression transmurale pratiquement nulle et qui devrait même être logiquement négative au niveau des voies de drainage. 